\title{
Lipid-based formulations in cosmeceuticals and biopharmaceuticals
}

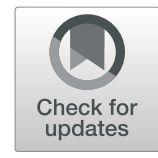

Anas Ahmad ${ }^{1,2}$ and Haseeb Ahsan ${ }^{3^{*}}$

\begin{abstract}
The fatty acids containing plant oils have been used as components of cosmetic formulations, and the best cosmetic function has been established for unsaturated fatty acids present in triglycerides. Many oils, fats, waxes, and phospholipids are employed as excipients in cosmetics and personal care products. Antioxidants such as carotenoids, retinoids, and tocopherols are used for their antioxidant properties because of their importance in therapeutic care. For skin care, the most important are oils with high contents of linoleic and a-linolenic acid which reduce the formation of eczemas or atopic dermatitis. These essential fatty acids incorporate into the cell membranes and regenerate the damaged lipid barrier of epidermis and restrict water loss. The unsaturated fatty acids show prominent healing effects on skin inflammation and are used in various cosmetic products. Mineral oils and waxes are also used in several products due to their property of skin tolerance, protection, cleansing, and viscosity. As cosmeceutical formulation, the nanoformulations are effective against irritated and inflamed skin and effective for therapy of atopic dermatitis and psoriasis. The lipid nanoparticles offer advantages of chemical stability and good skin compatibility that can be used for protection against radiation and aging.
\end{abstract}

Keywords: Cosmetics, Lipids, Fatty acids, Liposomes, Waxes, Lipid nanoparticles

\section{Introduction}

Humans have used cosmetics since early civilizations, and the products were obtained from natural compounds (milk, flowers, fruits, seeds, vegetables, etc.) and minerals (clay, ash, etc.). The cosmetic products have been used to clean, perfume, prevent body odor, and keep and protect the skin and teeth in good condition. Cosmetics are defined as any preparation intended to be used on the body for cleansing, perfuming, beautifying, odorant, and promoting well-being without affecting the structure or function of the body. Cosmetic refers to all products and substances used for cleansing the body and skin to make it more attractive and beautiful. The intention of using cosmetics is to protect the human body from the harmful effects of environment, process of aging, and maintaining and changing the odor and appearance of the body (Ahsan 2019; Siti Zulaikha et al.

\footnotetext{
* Correspondence: hahsan@jmi.ac.in; drhahsan@gmail.com

${ }^{3}$ Faculty of Dentistry, Department of Biochemistry, Jamia Millia Islamia (A

Central University), Jamia Nagar, New Delhi 110025, India

Full list of author information is available at the end of the article
}

2015). The ingredients in fragrances, humectants, emollients, polishes, lotions, creams, etc. are obtained from natural and chemical sources in cosmetics and personal care products (PCPs). Cosmetic preparations that influence the function and structure of the human skin such as sunscreens or antidandruff shampoos are considered as drugs. However, the added chemicals in the form of preservatives and fragrances may be hazardous and are sometimes prohibited due to health concerns (Ahsan 2019; Siti Zulaikha et al. 2015). Therefore, there is an increasing demand and awareness to the use of ingredients from natural sources such as biomolecules and macromolecules from plants, animals, and marine sources. The beneficial effects promoted from the use of lipid-rich substances into the formulations of various PCPs are considered as useful ingredients (Ahsan 2019). Hence, this review article attempts to identify the various complex lipids and derivatives found in cosmetic products which may have an important role on the body particularly skin. Kligman (2000) introduced the term "cosmeceutical" for cosmetic products that are applied for

(c) The Author(s). 2020 Open Access This article is licensed under a Creative Commons Attribution 4.0 International License, which permits use, sharing, adaptation, distribution and reproduction in any medium or format, as long as you give appropriate credit to the original author(s) and the source, provide a link to the Creative Commons licence, and indicate if changes were made. The images or other third party material in this article are included in the article's Creative Commons licence, unless indicated otherwise in a credit line to the material. If material is not included in the article's Creative Commons licence and your intended use is not permitted by statutory regulation or exceeds the permitted use, you will need to obtain permission directly from the copyright holder. To view a copy of this licence, visit http://creativecommons.org/licenses/by/4.0/. 
personal care having a combination of cosmetic and pharmaceutical applications. The word "cosmeceutical" derived from cosmetics and pharmaceuticals signifies that the product contains ingredients is intended for either personal and/or therapeutic use. Cosmeceuticals contain natural ingredients in products such as creams, lotions, ointments, and ingestible beauty products in the form of liquids, pills, and functional foods. These products contain ingredient or nutrients believed to promote healthy skin, hair, and nails and may include vitamins, minerals, plant extracts, and antioxidants (Ahsan 2019; Kim 2014; Ruocco et al. 2016). Marine sources have also shown potential as a rich source of biologically active compounds with cosmetic potential. The marine environment contains compounds such as polyunsaturated fatty acids (PUFA), polysaccharides, essential minerals and vitamins, antioxidants, enzymes, and bioactive peptides (Thomas and Kim 2013). Moreover, there is growing interest and potential toward bioactive compounds in cosmetics from natural resources because of their numerous health benefits and applications (Ahsan 2019).

Lipids are a diverse and distinct group of natural organic compounds found in plants, animals, and microorganisms. They comprise one of the three large classes of food products including proteins and carbohydrates consumed by animals (Fahy et al. 2005). Lipids (Greek "lipos") are natural compounds which include fatty acids and their derivatives and play a key role in the structure and metabolism of all living organisms. They are the major constituent of cell membrane structure (phospholipids and cholesterol derivatives) and serve as an important source and storage reserve of energy (triglycerides) (Fahy et al. 2005). The lipids are superior to carbohydrates for storage because the carbons chain of lipids are in a highly reduced state, which maximizes the energy released during digestion and oxidation into carbon dioxide and water (Fahy et al. 2005). They are hydrophobic since their structure contains long hydrocarbon chains and sometimes amphiphilic due to the presence of hydrophilic domains such as phosphates, glycerol, nitrogen, and alcohol and are insoluble in water but soluble in organic solvents (Fahy et al. 2005). The complex polar lipids are characterized by the presence of non-lipid chemical groups such as phosphate and carbohydrate moieties which are important formulations in cosmetics due to their amphipathic nature. Complex lipids may be classified into two groups based on their polarity into neutral lipids and polar lipids. The latter group includes lipids containing phosphate moiety known as phospholipids and the glucose containing glycolipids (Fig. 1-d) (Traversier et al. 2018). The classification of lipids is based on the International Union of Pure and Applied Chemistry (IUPAC) nomenclature (Fahy et al. 2005). Moreover, IUPAC celebrated its hundredth anniversary last year, i.e., 2019.

\section{Cosmetic fatty acids and lipids}

The lipids present in cosmetics intended to be applied to human skin to protect and improve the appearance of body, form a protective barrier on the skin, protect from external harmful substances and help to keep it hydrated and soft. The fatty acids commonly used as components of cosmetic formulations are a mixture of triglycerides of saturated and unsaturated fatty acids, i.e., they are esters of glycerol and higher fatty acids, containing even number of aliphatic carbon atoms (Bonnet 2018; Bialek et al. 2016) (Table 1). In cosmetic emulsions which account for the bulk of personal care products, the major ingredients present are lipids (plant oils, fatty acids, etc.) or their derivatives (emollients, emulsifiers). In recent years, the complex lipids used in cosmetic formulations are often obtained from plant or biotechnology origin, making it possible to promote oilseed crops and develop new extraction techniques and methods (Bonnet 2018). Fatty acids (FAs) are important in maintaining the structure and function of the outer layer or epidermis (stratum corneum, SC) which contains glycolipids, intercellular lipids (cement), and a lipid coat of the skin called the natural moisturizing factor (NMF). Lipids in intercellular matrix connect the SC, ensuring its cohesiveness, ability to protect the skin from xenobiotics, and forming a barrier against water loss (Bialek et al. 2016). The major fatty acids present in cosmetics are unsaturated fatty acids in triglycerides (TGs), in particular the EFAs linoleic acid (omega-6) and $\alpha$-linolenic acid (omega3 ). For skin care, the most important are the oils with high content of omega- 6 and omega- 3 that prevent the formation of eczemas (Bialek et al. 2016). The lipids present in epidermis are generally ceramides (sphingolipids) and essential fatty acids (EFAs). Linoleic acid (LA) is an essential constituent of EFAs in ceramides which decrease in the epidermis with age causing skin sensitivity and roughness. The epithelial cells (keratinocytes) constituting a major part of epidermis do not produce vitamin D (D6, D5), and the conversion of LA and alpha-linolenic acid (ALA) does not occur. Therefore, the topical application of EFAs on skin in the form PCP is important for the functions and appearance of skin. The hydrating and softening properties of skin and hair are achieved by using plant seed oils rich in FAs, which reduce transepidermal water loss (TEWL) (Bialek et al. 2016).

The oils derived from plants are generally regarded as healthier as compared to animal fats due to their beneficial FAs. There has been an increasing interest in the use of oils obtained from nuts (almonds, pistachios, walnuts), plants (borage, primrose, perilla), fruits seeds (cherry, berries), and vegetables (carrot), for direct consumption as well as for the manufacture of PCP (Bialek et al. 2016). Vegetable oils have been successfully used in cosmetics due to their softening and smoothing 


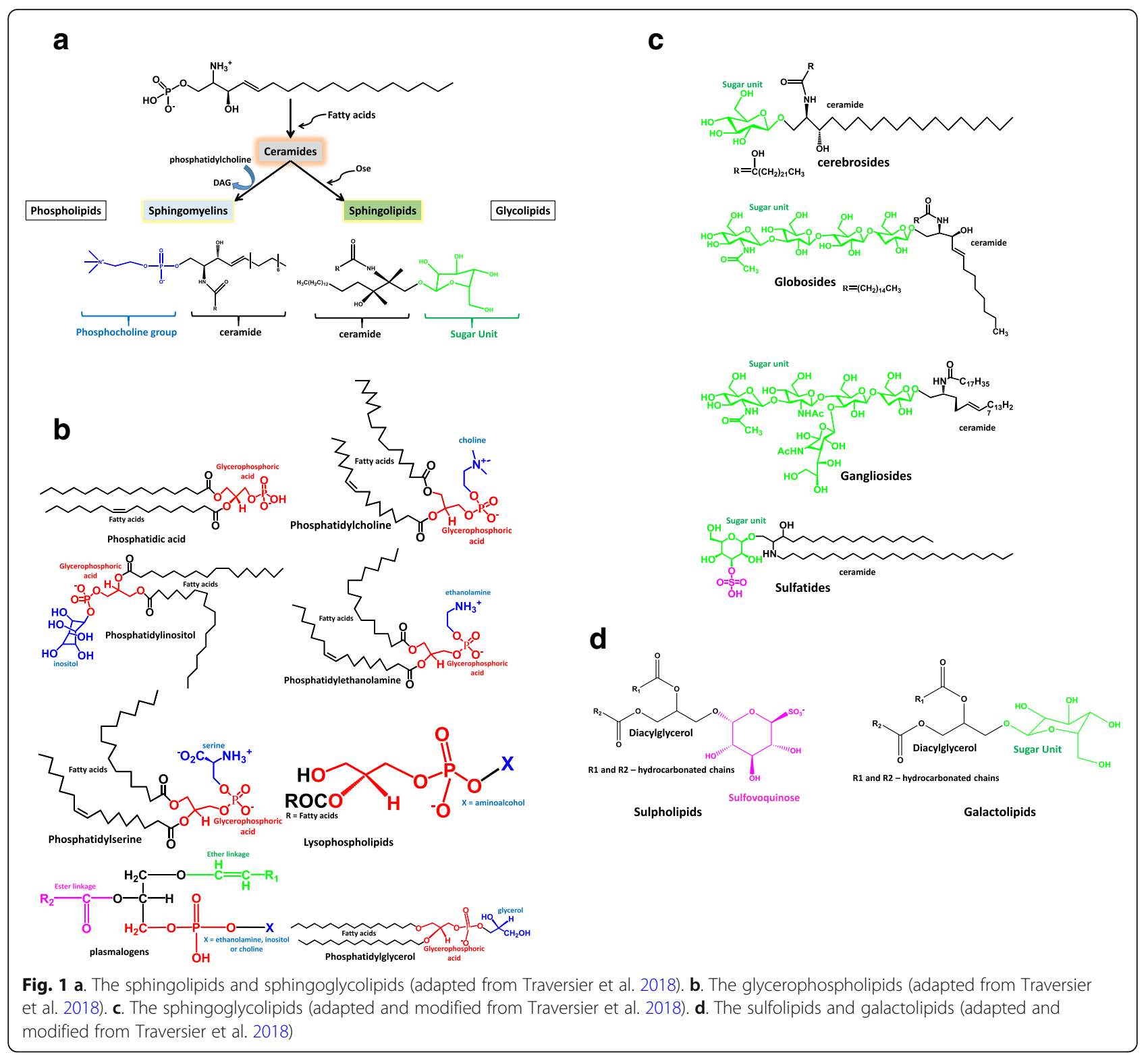

properties for which they are classified as emollients. They prevent water loss forming a protective layer on the epidermis softening the SC and reducing the inflammation of the skin. Moreover, they play an important physiological role in the body mainly in the synthesis of eicosanoids or "local hormones" (prostaglandins, prostacyclins, thromboxanes, and leukotrienes). Hence, the deficiency of fatty acids and lipids significantly affects vascular fragility, reduces immune functions, interferes with clotting process, and increases the chance of forming atherosclerosis (Bialek et al. 2016; Zielinska and Nowak 2014). Moreover, the oils incorporate into the cell membranes and regenerate the damaged lipid barrier of epidermis restricting water loss. The unsaturated fatty acids show pronounced healing effects on dermatoses such as atopic skin inflammation and are used in creams, emulsions, cosmetic milks, ointments, hair conditioners, cosmetic masks, lipsticks, bath fluids, nail polishes, etc. Therefore, the deficiency of fatty acids may result in excessive dryness of the skin leading to various

Table 1 The use of plant vegetable oils in cosmetic products (adapted and modified from Alvarez and Rodríguez, 2000)

\begin{tabular}{ll}
\hline Facial care & Hair care \\
\hline $\begin{array}{l}\text { Almond, apricot, hazelnut, borage, jojoba, avocado, olive, wheat germ, macadamia, } \\
\text { grapeseed }\end{array}$ & $\begin{array}{l}\text { Almond, borage, avocado, cocoa butter, jojoba, sesame, } \\
\text { macadamia }\end{array}$ \\
\hline
\end{tabular}


health and medical concerns. Hence, the use of lipids as cosmetics ingredients illustrates their importance for skin and hair care (Zielinska and Nowak 2014; Alvarez and Rodriguez 2000).

\section{Waxes}

Waxes are formed by the combination of fatty acid esters with high molecular weight monohydroxy alcohols that are hard brittle substances. Waxes are present in both plants and animals including marine life. They minimize water loss in plants and act as waterproofing agents in animals. Liquid wax (esters) from jojoba seeds and sperm whale oil is used in various commercial cosmetic products as lubricants, wax polishes, and additives (Alvarez and Rodriguez 2000). Wax such as lanolin, a complex mixture of fatty acid esters and high molecular weight aliphatic and steroid alcohol, is obtained from the sebaceous secretions (wool fat) of sheep (Ovis aries). The sebaceous glands secrete an oily and waxy substance known as sebum. It protects and moisturizes the skin and hair, but it may be depleted by chemicals, pollutants, and sun and aging process, resulting in dry skin and hair. It has strong emulsifying properties and is used as a binder, stabilizer, and conditioner in products such as lipsticks, make-up and eye products, creams, and lotions. It is an odorless and tasteless wax lipid which is soluble in mineral oils making it useful in bath oil products (Alvarez and Rodriguez, 2000).

Jojoba oil, a mixture of long chain, linear liquid wax esters extracted from the seeds of the desert shrub Simmondsia chinensis, provides oxidative stability, acts as an emollient and effective moisturizer for hair and skin. It replenishes lost skin and hair and maintains the natural $\mathrm{pH}$ balance. It also reduces wrinkles, psoriasis, acne, and neurodermatitis and restores the skin to its normal vitality and health. The addition of jojoba oil in products improves the viscosity of lubricants and it remained stable when heated at high temperature (Alvarez and Rodriguez 2000). Bees wax is secreted from the glands of Apis mellifera, and it imparts outstanding properties to bodycare products and is used as an excipient in formulations to increase the viscosity and consistency of preparation (Alvarez and Rodriguez 2000). It is added to bar soaps to make them harder and is also used in creams, lotions, and lip balms. Spermaceti, a wax obtained from the head of sperm whale (Physeter macrocephalus), consists of cetylpalmitate, cetylic alcohol, and other esters that contain fatty acids and alcohols. It is the lipid that is employed to give consistency and texture to cerates and ointments (Alvarez and Rodriguez 2000). The lanolin wax is odorless and tasteless and is used primarily in lipsticks and glosses. It has water absorption capability and mineral oil solubility and is used in lipsticks, moisturizers, creams and lotions, cleansing products, and emulsions. It is useful in water-resistant products such as sunscreens, baby products, and hand and body creams. Therefore, waxes are used as solubilizers in sunscreens, perfumes, pharmaceuticals, hair products, shampoos, soaps, dishwashing detergents, shaving lotions, astringents, and colognes (Alvarez and Rodriguez 2000).

\section{Essential and mineral oils}

Essential oils and their compounds are widely used in cosmetics as they offer a variety of benefits including pleasant aroma. Their useful biological activities include pathophysiological activity such as analgesic, antiseptic, antimicrobial, carminative, diuretic, spasmolytic, hyperemic, and stimulatory properties. Due to the antimicrobial and antifungal properties of essential oils, PCP does not generally require a chemical preservative if they contain an essential oil as an active ingredient (e.g., rosemary oil, eucalyptus oil). Essential oils generally consist of lipophilic, small, and non-polar molecules such as terpenoids, rosemary oil, etc. However, apart from their positive effects, the essential oils and fragrances can also be a source of potential allergic reactions (Sarkic and Stappen 2018; Oliver et al. 2015). Biochemically, mineral oils and waxes are mixtures of predominantly saturated hydrocarbons consisting of straight-chain, branched, and ring structures with carbon chain length greater than C16. The lipids used in PCPs are highly refined and of the highest purity since the manufacturing process is designed to exclude potentially carcinogenic substances like polycyclic hydrocarbons (PAH) that may be present in the starting crude mineral oils. The mineral oils and waxes currently used in cosmetic products are complex combination of hydrocarbons obtained from petroleum fractions with carbon chain length ranging from C18-C90 (Weber et al. 2018; Lachenmeier et al. 2017). Mineral oils and waxes used in European cosmetic products are of pharmaceutical grade within the specifications of European Pharmacopeia and in compliance with the European Union's cosmetic products regulations (Petry et al. 2017; Chuberre 2019). The human exposure to mineral oils in cosmetics is mainly through topical application, largely depends on the oil's ability to penetrate the skin. The various studies done conclude that these lipids used in cosmetics do not penetrate the skin layers and remain in the SC. Nevertheless, given their lack of dermal uptake and systemic availability, mineral oils and waxes are unlikely to represent a major systemic human health risk even after repeated long-term exposure (Petry et al. 2017).

\section{Liposomes}

Liposomes are the small artificial spherical vesicles that can be created from a wide assortment of amphiphilic molecules both positive and negative charged, including cholesterol and natural phospholipids which determine 
their rigidity or fluidity and are promising carriers for drug delivery (Moore 2002; Akbarzadeh et al. 2013). The liposomal membrane is generally made up of synthetic or natural phospholipids where cholesterol incorporation enhances the stability. As a result, the physicochemical characteristics of liposomes are majorly regulated by the incorporated phospholipid. Lecithins are the majorly employed phospholipids in the liposomes which are either derived from natural sources like eggs and soybeans etc., or are of synthetic character (Soni et al. 2016). They may be particularly promising as intracellular delivery agents for ribosomes, proteins or peptides, and DNA. Liposomes are extensively used as carriers for molecules in cosmetic and pharmaceutical industries for delivery systems that entrap unstable compounds such as antibiotics, antioxidants, and biomolecules. They can enclose both hydrophobic and hydrophilic compounds, avoiding the decomposition of the entrapped molecules and releasing them to designated targets. The unsaturated phosphatidylcholines (PC) (egg or soybean phosphatidylcholine) form rather permeable and unstable bilayers, whereas the saturated phospholipids with acyl chains (dipalmitoylphos phatidylcholine) form more rigid and impermeable structures (Akbarzadeh et al. 2013). When skin is affected by eczema or gets damaged, empty liposomes interact with skin macromolecules helping in the rejuvenation of skin and making the SC perform its defensive functions. When they are used as carriers for delivery of molecules, they enhance the penetration, solubility and stability, targeting the ingredients to their desired site of action, reducing toxicity, and increasing pharmacokinetics (Ahmadi Ashtiani et al. 2016).

Different types of cosmetic liposomes have been formulated and characterized based upon the feature of cosmeceutical agents. These can be transfersomes of 200-300 nm diameter, extremely robust, having sound efficacy, readily penetrate the skin surface, and cross the skin's corneal layer, intracellular and transcellular passages. These are generally formulated with phospholipids and cholesterol along with surfactants (Sharma et al. 2018). The other category is of niosomes which are vesicles of 100-1000 nm, made up of polyglycerols, and are very effective in improving the performance of cosmeceutical agents and skin care products by improving the skin penetration. There are other types of liposomes like ultrasomes, ethosomes, and photosomes which are named based upon their one or more peculiar physicochemical, morphological, or functional characteristics (Costa and Santos 2017). The other criteria for classification of liposomes based upon lamellar structure and size like small liposomes of size 10-100 nm, larger single layered liposomes of up to $3 \mu \mathrm{m}$ and multi-layered liposomes consisting of more than $3 \mu \mathrm{m}$. The liposomes consist of a hydrophobic core but can be adapted to carry the payload of all kinds of molecules being hydrophobic, hydrophilic, or amphiphilic. The drug loading capacity of liposomes and encapsulation efficiency of the active payload depends upon several factors like the amount of lipid, the diameter of the extruding syringe, and flow rates of the aqueous and organic phases. Some recent liposomal formulations for cosmeceutical applications include nanosomes, carrying pro-retinol $\mathrm{A}$ as the active molecule and employed as anti-wrinkle agent, available by the trade name Revitalift (L'Oreal). Other significant applications of liposomal cosmetic formulations include anti-aging, antioxidants, revitalizing agents, stimulating the production of skin collagen, and antiinflammatory agents (Hatem et al. 2018).

Despite possessing a large number of significant properties like high drug loading capacity, enhanced encapsulation efficiency, prolonged stability, desirable and controlled particle size and narrow particle size distribution and their diffusability up to deeper skin layers, and their overall great capability in delivering active ingredients in cosmeceuticals, liposomes possess many challenges in their formulation and applicability. Sometimes their physical and chemical stability, particle size, and particle size distribution may not serve to be long-term characteristic especially in the regions with large temperature variations (van Tran et al. 2019). As cosmeceutical agents, liposomes have been employed in various dermatological conditions like acne vulgaris and hyperpigmentation formulated with clindamycin hydrochloride (clindamycin $\mathrm{HCl}$ ) and 4-n-butylresorcinol (BR), respectively (Rahimpour Y and Hamishehkar 2012). So the lipid based particulate systems were introduced first in the early 90s and have greatly evolved with time form the preliminary liposomal formulations to the recent more advanced nanosystems like solid lipid nanoparticles and nanolipid carriers, where the limitations of liposomes like burst release of active cosmeceutical, solubility limitations, high production costs, and probable oxidation and hydrolytic reactions of phospholipids have greatly been overcome (Antunes et al. 2017).

\section{Lipid nanoparticles}

During recent times, a large number of studies have been undertaken depicting the use of lipid based materials for topical applications. The materials include but not limited to emulsions, microemulsions, multiple emulsions, etc. Sometimes, some formulations appear in the market with trade names suggesting the nanomaterials' activity but such activity could be barely proven scientifically (Garces et al. 2018). Solid lipid nanoparticles (SLN) and nanostructured lipid carriers (NLC) are nanoparticles composed of solid lipid or solid lipid blend, respectively. Among these two, solid lipid nanoparticles 


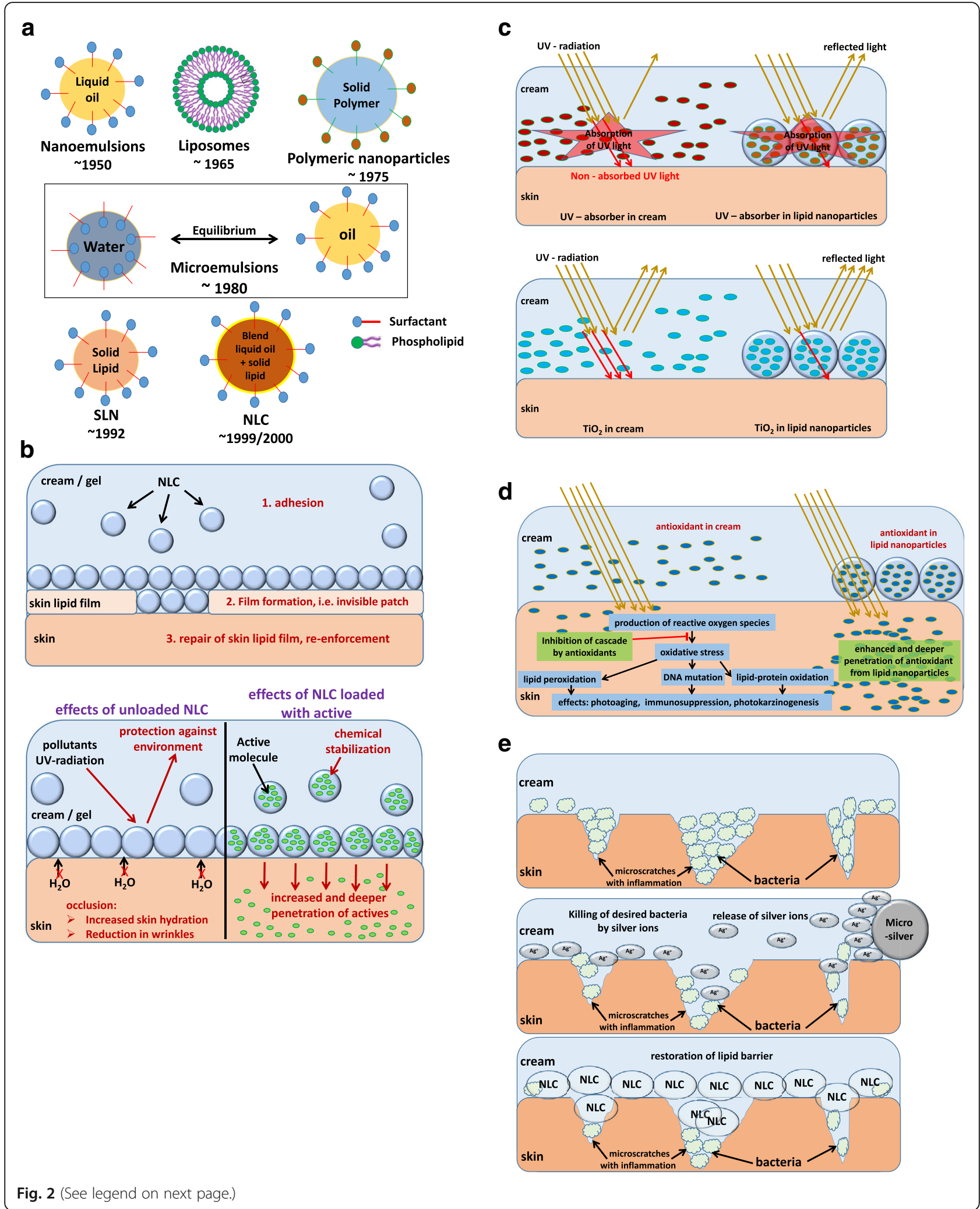


(See figure on previous page.)

Fig. 2 a. The development of dermal nanocarrier systems from nanoemulsions to solid lipid nanoparticles (SLN) and nanostructured lipid carriers (NLC) (adapted from Muller et al. 2014) with permission. b. The effect of lipid nanoparticles (LN) on the skin. The LN adhere and form a film on the skin which protects against environmental hazards and increases dermal penetration (adapted from Muller et al. 2014) with permission. c. The incorporation of sunscreens into NLCs increases their UV absorption capacity, in addition, UV is scattered by NLC particles; TiO2 nanoparticles can be firmly enclosed in larger NLCs eliminating skin penetration and also increasing the UV scattering effect (adapted from Muller et al., 2014) with permission. $\mathbf{d}$. Effect of radiation on the skin leading to oxidative stress through ROS. The antioxidants from NLCs can more efficiently block the oxidative stress cascade (adapted from Muller et al. 2014) with permission. e. Irritated inflamed skin with microscratches and bacterial colonization (upper), antibacterial effect of silver ions released from microsilver (middle), and restoration of skin barrier by adsorbing highly adhesive NLC film (adapted and modified from Muller et al. 2014 with permission)

were released first into the market and hence described as first generation lipid nanoparticles and were followed by improved second generation nanoparticles, i.e., nanostructured lipid carriers (Chen et al. 2017). These nanoparticles generally function as nanocarrier of medicinally active agents like drugs, inhibitors, and other pharmaceutical or cosmeceutical agents (Kaul et al. 2018; Aziz et al. 2019). These are synthesized and formulated by high-pressure homogenization and characterized for their physicochemical and morphological characteristics by a number of techniques like transmission electron microscopy, scanning electron microscopy, and atomic force microscopy for their shape, size, and surface morphology topographical features (Ahmad et al. 2019). They combine the properties of various traditional nanocarriers, e.g., chemical stabilization, good skin compatibility, and used for protection against UV and IR radiation in anti-aging products. As cosmeceutical formulation, the silver-nanolipid (sNLC) complex is effective in skin care for the therapy of atopic dermatitis and psoriasis. The SLN are composed of a lipid matrix being solid at room temperature, and in NLCs, it is a blend of

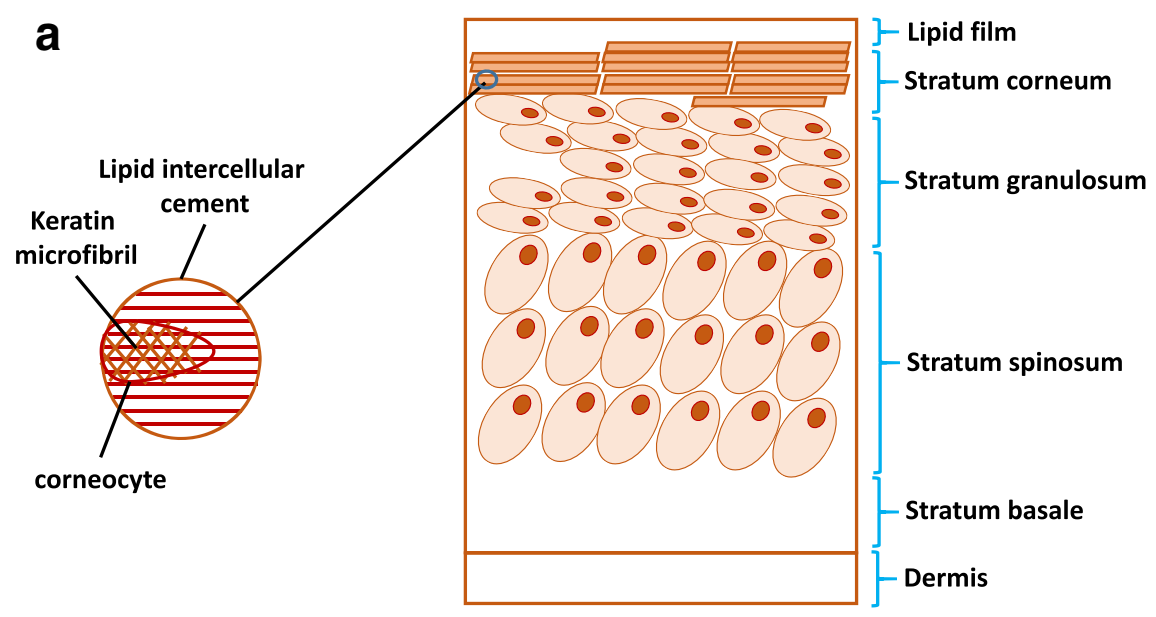

b

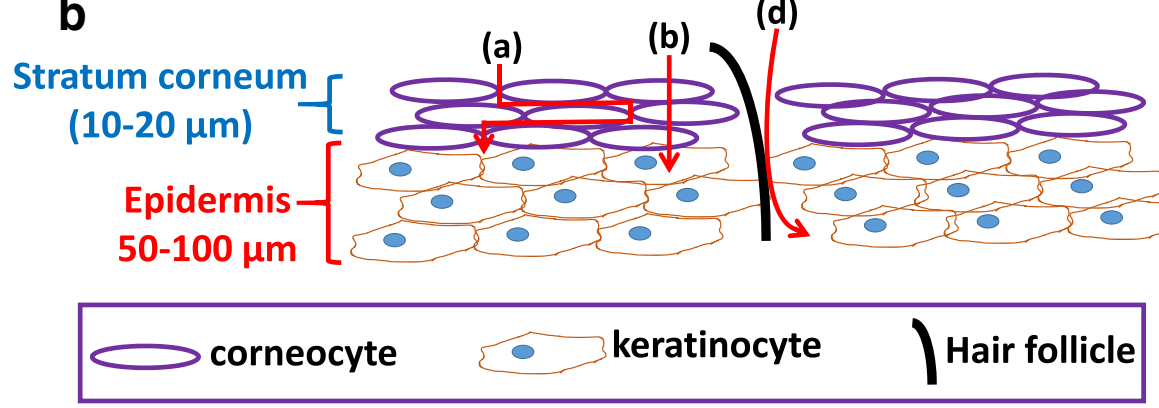

Fig. 3 a. The barrier functions of the skin are performed by the lipids present in the outermost layer of the skin, the stratum corneum (adapted from Traversier et al. 2018). b. The application and target of cosmetic products on the outer layers of skin. The diagram shows the probable routes of transport of therapeutic products through the skin (a) intercellular through lipid bilayers, (b) transcellular through keratinocytes (c) shunt through hair follicles and sweat glands. The stratum corneum (SC) is the outermost layer of the skin consisting of mostly dead skin cells is a major barrier to harmful substances, and the epidermis is the major outer thin layer of skin (adapted and modified from Zakrewsky et al. 2015 with permission) 
oils and solid lipids (Fig. 2a-e). The lipid nanoparticles are composed of lipids (e.g., cetyl palmitate) and stabilizers (e.g., alkyl polyglycosides) which can be produced on a biotechnological scale. Due to their lipidic nature, the lipid nanoparticles are ideally suited to incorporate lipophilic molecules and poorly water soluble peptides. Hence, the lipid nanoparticles can deliver dermal products more efficiently than the traditional nanocarriers due to their solubility and stability (Müller et al. 2016; Muller et al. 2014). Apart from drug delivery purpose, a number of lipid-based nanomaterials have been used in the recent past for varied purposes. Recently, lipid nanocarriers having size $100 \mathrm{~nm}$ or less have particularly attracted the attention as agents for therapeutic improvement of medicinal agents. The traditional cosmeceuticals like emulsions and creams are being largely replaced by solid lipid nanoparticles (SLN) and nanostructured lipid carriers (NLCs) (Janfaza and Razavi 2017). The cosmeceutical compounds are imparted the advantages like enhanced stabilization, sustained and controlled release, better adhesion and occlusion properties, and robust film formation on skin. Also, enhancement in the loading capacity, ease in the potential supersaturated topical formation, and large-scale production are additional benefits associated with lipidbased nanomaterials. The cosmeceutical agents are imparted with the skin penetration enhancement a better skin hydration along with the excellent tolerability of lipid materials. For example, incorporation of solid lipid nanocarriers to a traditional cream enhances the skin hydration for prolonged periods. Additionally, there are reports where solid lipid nanocarriers have been employed as physical sunscreen agents and carriers of molecular sunscreens (Khezri et al. 2018).

Nanosized particulate materials exhibit adhesion when in contact with the skin surface. This property is an integral part of lipid-based nanoparticles and liposomes. It is reported that in a specific proportion, lipid nanoparticles of $200 \mathrm{~nm}$ are capable of forming a monolayer when a fixed concentration of these is applied. This monolayer being hydrophobic has the capability of forming a moisture barrier over skin, reducing the loss of water caused by evaporation. This property of lipid-based nanomaterial is employed to control the occlusion and hydration paradigms (Naseri et al. 2015). The lipid-based nanocarriers like solid lipid nanoparticles have been used as the carriers of chemically labile agents like vitamin E so as to protect the agent from chemical degradation (Suter et al. 2016). The characteristic of lipid-based nanomaterials is that they can be incorporated into aqueous dispersions and remain physically stable and also can be readily mixed with dermal formulations like cream and can form an adhesive film providing an occlusive effect. This occlusive effect elevates the penetration of active cosmeceuticals into the skin thereby enhancing its effects (Müller et al. 2016; Muller et al. 2014).

\section{Dermatopathology of lipid cosmeceuticals}

Two major functions of SC are to prevent excessive water loss and prevent penetration of xenobiotics into the dermal layers to avoid an immune response. The main components of the SC are the corneocytes and intercellular lipids (Fig. 3a, b) which are included in an envelope of highly crosslinked proteins (loricrin, involucrin, filaggrin). The optimal water gradient is maintained by corneocyte lipids and the NMF which are responsible for keeping adequate hydration of SC (Müller et al. 2016; Muller et al. 2014). Most of the lipids constituting the SC are ceramides, cholesterol and fatty acids, and a minor component consisting as phospholipids and cholesterol sulfate. The PC fractions from soybean oil participate in improving the skin humidity and emollient properties; the phosphatidyl lecithins also provide softening properties in creams, lotions, and shampoos. In addition to the known functional applications like surfactants or emulsifiers, the polar lipids also play an important role in various physiological functions to keep the skin healthy and disease free (Traversier et al. 2018).

\section{Conclusion}

The demand for active, natural, safe, cosmetic ingredients including lipids is large and constantly increasing. Many oils, fats, waxes, and phospholipids are employed as excipients in cosmetic and PCP formulations. Antioxidants such as carotenoids, retinoids, and tocopherols are used for their antioxidant properties because of their importance in therapeutic care. Moreover, lipid nanoparticles can be used for the protection against harmful radiations and as anti-aging compounds. As cosmeceutical formulation, the nanoformulations are effective against irritated and inflamed skin and effective for therapy of atopic dermatitis and psoriasis.

\section{Abbreviations}

EFAs: Essential fatty acids; FAs: Fatty acids; MOAH: Mineral oil aromatic hydrocarbons; NLC: Nanostructured lipid carriers; NMF: Natural moisturizing factor; PCP: Personal care products; PUFA: Polyunsaturated fatty acids; SLN: Solid lipid nanoparticles; TEWL: Transepidermal water loss

\footnotetext{
Acknowledgements

This review article is dedicated to the memory of my late beloved father. He was the greatest father and husband, the finest gentleman, and an extraordinary human being. HA wishes to that thank those authors from whose work the various data/tables/figures have been used and adapted. AA would like to thank Intitute of Nano Science and Technology (INST) Mohali, for providing a Senior Reserch Fellowship. HA also wishes to thank Prof. Fahim H. Khan and Prof. Waseem A. Siddiqui for their motivation, encouragement, and inspiration. The various cosmetic and pharmaceutical companies such as L'Oreal, Avon, Olay Regenerist, and Este'e Lauder are the copyright owners of the products available in the market, and they have been acknowledged in the review article wherever possible. The authors wish to acknowledge the Editor for his thoughtful and encouraging comments.
} 


\section{Authors' contributions}

AA and HA wrote and revised the manuscript. HA conceived the idea for review. The author(s) read and approved the final manuscript.

\section{Funding}

None

\section{Availability of data and materials}

Not applicable

\section{Ethics approval and consent to participate}

Not applicable

\section{Consent for publication}

Not applicable/approval wherever applicable

\section{Competing interests}

The authors declare that they have no competing interests.

\section{Author details}

'Department of Pharmacology and Toxicology, National Institute of Pharmaceutical Education and Research (NIPER), Sector 67, Mohali, Punjab 160062, India. ${ }^{2}$ Department of Nano-Therapeutics, Institute of Nano Science and Technology, Habitat Centre, Phase - 10, Sector 64, Mohali, Punjab 160062, India. ${ }^{3}$ Faculty of Dentistry, Department of Biochemistry, Jamia Millia Islamia (A Central University), Jamia Nagar, New Delhi 110025, India.

\section{Received: 25 November 2019 Accepted: 28 February 2020}

Published online: 06 May 2020

\section{References}

Ahmad A, Khan F, Mishra RK, Khan R. Precision cancer nanotherapy: evolving role of multifunctional nanoparticles for cancer active targeting. J. Med. Chem. 2019. https://doi.org/10.1021/acs.jmedchem.9b00511.

Ahmadi Ashtiani HR, Bishe P, Lashgari N, Nilforoushzadeh MA, Zare S, et al. Liposomes in cosmetics. J Skin Stem Cell. 2016;3(3):e65815.

Ahsan H. The biomolecules of beauty: biochemical pharmacology and immunotoxicology of cosmeceuticals. J Immunoassay Immunochem. 2019:40:91-108.

Akbarzadeh A, Rezaei-Sadabady R, Davaran S, Joo SW, Zarghami N, Hanifehpour Y, Samiei M, Kouhi M, Nejati-Koshki K. Liposome: classification, preparation, and applications. Nanoscale Res Lett. 2013;8(1):102.

Alvarez AMR, Rodríguez MLG. Lipids in pharmaceutical and cosmetic preparations. Grasas y Aceites (Espanol). Int J Fats Oils. 2000;51(1-2):74-96.

Antunes A, Pereira P, Reis C, Rijo P, Reis C. Nanosystems for skin delivery: from drugs to cosmetics. Curr Drug Metab. 2017;18(5):412-25.

Aziz ZAA, Mohd-Nasir H, Ahmad A, Mohd SSH, Peng WL, Chuo SC, Khatoon A, Umar K, Yaqoob AA, Mohamad Ibrahim MN. Role of nanotechnology for design and development of cosmeceutical: application in makeup and skin care. Front. Chem. 2019;7:739. https://doi.org/10.3389/fchem.2019.00739.

Bialek A, Bialek M, Jelinska M, Tokarz A. Fatty acid profile of new promising unconventional plant oils for cosmetic use. Int J Cosmet Sci. 2016;38(4):382-8,

Bonnet C. Lipids, a natural raw material at the heart of cosmetics innovation. Oilseeds Fats Crops Lipids. 2018;25(5):D501. https://doi.org/10 $1051 / \mathrm{ocl} / 2018055$

Chen J, Wei N, Lopez-Garcia M, Ambrose D, Lee J, Annelin C, Peterson T. Development and evaluation of resveratrol, vitamin $\mathrm{E}$, and epigallocatechin gallate loaded lipid nanoparticles for skin care applications. Eur J Pharm Biopharm. 2017:117:286-91.

Chuberre B, Araviiskaia E, Bieber T, Barbaud A. Mineral oils and waxes in cosmetics: an overview mainly based on the current European regulations and the safety profile of these compounds. J Eur Acad Dermatol Venereol. 2019;33(Suppl 7):5-14. https://doi.org/10.1111/jdv.15946.

Costa R, Santos L. Delivery systems for cosmetics - from manufacturing to the skin of natural antioxidants. Powder Tech. 2017;322:402-16.

Fahy E, Subramaniam S, Brown HA, Glass CK, Merrill Jr AH, Murphy RC, Raetz CR, Russell DW, Seyama Y, Shaw W, Shimizu T. A comprehensive classification system for lipids. Eur J Lipid Sci Technol. 2005:107(5):337-64.

Garcês A, Amaral MH, Lobo JS, Silva AC. Formulations based on solid lipid nanoparticles (SLN) and nanostructured lipid carriers (NLC) for cutaneous use: a review. Eur J Pharm Sci. 2018;112:159-67.
Hatem S, Nasr M, Elkheshen SA, Geneidi AS. Recent advances in antioxidant cosmeceutical topical delivery. Curr Drug Delivery. 2018;15(7):953-64.

Janfaza S, Razavi S. Lipid-Based Nanoformulations for treatment of skin diseases. Cham: Nanotechnology applied to pharmaceutical technology Springer; 2017. p. 247-66.

Kaul S, Gulati N, Verma D, Mukherjee S, Nagaich U. Role of nanotechnology in cosmeceuticals: a review of recent advances. J Pharm (Cairo). 2018;2018: 3420204. https://doi.org/10.1155/2018/3420204.

Khezri K, Saeedi M, Dizaj SM. Application of nanoparticles in percutaneous delivery of active ingredients in cosmetic preparations. Biomed Pharmacother. 2018:106:1499-505.

Kim SK. Marine cosmeceuticals. J Cosmet Dermatol. 2014;13(1):56-67.

Kligman AM. Cosmetics a dermatologists looks to the future: promises and problems. Dermatol. Clin. 2000;18:699-709.

Lachenmeier DW, Mildau G, Rullmann A, Marx G, Walch SG, Hartwig A, Kuballa T. Evaluation of mineral oil saturated hydrocarbons (MOSH) and mineral oil aromatic hydrocarbons $(\mathrm{MOAH})$ in pure mineral hydrocarbon-based cosmetics and cosmetic raw materials using $1 \mathrm{H}$ NMR spectroscopy. F1000Res. 2017:6:682. https://doi.org/10.12688/f1000research.11534.2.

Moore A. The biochemistry of beauty the science and pseudo-science of beautiful skin. EMBO reports. 2002;3(8):714-7.

Müller RH, Alexiev U, Sinambela P, Keck CM. Nanostructured lipid carriers (NLC): the second generation of solid lipid nanoparticles. Berlin, Heidelberg: Percutaneous penetration enhancers chemical methods in penetration enhancement Springer; 2016. p. 161-85.

Muller RH, Staufenbiel S, Keck CM. Lipid nanoparticles (SLN, NLC) for innovative consumer care \& household products. H\&PC Today-Household Personal Care Today. 2014;9(2):18-24

Naseri N, Valizadeh H, Zakeri-Milani P. Solid lipid nanoparticles and nanostructured lipid carriers: structure, preparation and application. Adv Pharm Bull. 2015;5(3):305.

Oliver B, Krishnan S, Rengifo PM, et al. Cosmeceutical contact dermatitis cautions to herbals. Curr Treat Options Allergy. 2015;2:307-21. https://doi. org/10.1007/s40521-015-0066-9.

Petry T, Bury D, Fautz R, Hauser M, Huber B, Markowetz A, Mishra S, Rettinger K, Schuh $W$, Teichert T. Review of data on the dermal penetration of mineral oils and waxes used in cosmetic applications. Toxicol Lett. 2017;280:70-8. https://doi.org/10.1016/j.toxlet.2017.07.899.

Rahimpour Y, Hamishehkar H. Liposomes in cosmeceutics. Expert Opin Drug Deliv. 2012;9(4):443-55. https://doi.org/10.1517/17425247.2012.666968.

Ruocco N, Costantini S, Guariniello S, Costantini M. Polysaccharides from the marine environment with pharmacological, cosmeceutical and nutraceutical potential. Molecules. 2016; 21(5). https://doi.org/10.3390/molecules21050551 pii: E551.

Sarkic A, Stappen I. Essential oils and their single compounds in cosmetics-a critical review. Cosmetics. 2018;5:11.

Sharma N, Singh S, Kanojia N, Grewal AS, Arora S. Nanotechnology: a modern contraption in cosmetics and dermatology. App Clin Res, Clin Trial Reg Affair. 2018:5(3):147-58.

Siti Zulaikha R, Sharifah Norkhadijah SI, Praveena SM. Hazardous ingredients in cosmetics and personal care products and health concern: a review. Public Health Research. 2015;5(1):7-15.

Soni V, Chandel S, Jain P, Asati S. Role of liposomal drug-delivery system in cosmetics. In Nanobiomaterials in galenic formulations and cosmetics. 2016; 10: William Andrew Publishing. pp. 93-120.

Suter F, Schmid D, Wandrey F, Zülli F. Heptapeptide-loaded solid lipid nanoparticles for cosmetic anti-aging applications. Eur J Pharm Biopharm. 2016;108:304-9.

Thomas NV, Kim SK. Beneficial effects of marine algal compounds in cosmeceuticals. Mar Drugs. 2013;11(1):146-64.

Traversier M, Gaslondes T, Milesi S, et al. Polar lipids in cosmetics: recent trends in extraction, separation, analysis and main applications. Phytochem Rev. 2018; 17:1179-210.

Van Tran V, Moon JY, Lee YC. Liposomes for delivery of antioxidants in cosmeceuticals: challenges and development strategies. I Controlled Release. 2019; [epub ahead of print].

Weber S, Schrag K, Mildau G, Kuballa T, Walch SG, Lachenmeier DW. Analytical methods for the determination of mineral oil saturated hydrocarbons (MOSH) and mineral oil aromatic hydrocarbons (MOAH) - a short review. Anal Chem Insights. 2018;13:1177390118777757. https://doi.org/10.1177/ 1177390118777757. 
Zakrewsky M, Kumar S, Mitragotri S. Nucleic acid delivery into skin for the treatment of skin disease: proofs-of-concept, potential impact, and remaining challenges. J Control Release. 2015;219:445-56.

Zielinska A, Nowak I. Fatty acids in vegetable oils and their importance in cosmetic industry. Chemik. 2014;68(2):103-10.

\section{Publisher's Note}

Springer Nature remains neutral with regard to jurisdictional claims in published maps and institutional affiliations.

Ready to submit your research? Choose BMC and benefit from:

- fast, convenient online submission

- thorough peer review by experienced researchers in your field

- rapid publication on acceptance

- support for research data, including large and complex data types

- gold Open Access which fosters wider collaboration and increased citations

- maximum visibility for your research: over $100 \mathrm{M}$ website views per year

At $B M C$, research is always in progress.

Learn more biomedcentral.com/submissions 